\title{
Diversity of over storey plant communities of tropical forest covers of Balasore district, Odisha, India
}

\begin{abstract}
The structure and function of a forest ecosystem is maintained by upper storey vegetation layer which principally consist of tree species. The tropical forest covers of Balasore, one of the coastal district of Odisha was analysed for structure, composition and diversity of upper storey vegetation layer $(\geq 30 \mathrm{~cm}$ circumference at breast height). A total of 94 tree species representing 77 genera and 38 families were recorded in this area. The average number of species per family was nearly equal to 2.5 and per genus was $>1.2$.The species diversity index and concentration dominance of the forest were 3.68 and 0.055 , respectively. The Importance Value Index (IVI) of species ranged from 0.55 to 40.47 . The estimated diversity indices indicated heterogeneity of the tropical forest covers of Balasore district in composition, structure and function. Thus rich over storey plant diversity with many of them as rare occurrence in the area supports the need of conservation for future use and sustenance.
\end{abstract}

Keywords: floristic composition, species diversity, concentration of dominance, IVI, conservation
Volume 8 Issue I - 2018

\author{
RK Mishra,' S Parhi, ${ }^{2}$ AK Biswal ${ }^{2}$ \\ 'Department of Wildlife and Biodiversity Conservation, North \\ Orissa University, Baripada, India \\ ${ }^{2}$ Department of Botany, North Orissa University, India
}

Correspondence: RK Mishra, Department of Wildlife and Biodiversity Conservation, North Orissa University, Takatpur, Baripada-757 003, India, Email rabikumishra@gmail.com

Received: February 05, 2016 | Published: January 09, 2018

\section{Introduction}

Vegetation diversity assessment in tropical forests have mostly been concentrated on tree species than other plant life forms, because tree species diversity is an important aspect of forest ecosystem structure and fundamental to tropical forest biodiversity. Tropical forests, the major repository of biodiversity, are undergoing rapid fragmentation and degradation all over the world. ${ }^{1}$ These covers $7 \%$ of the earth's land surface, but harbours more than half of the world's species $^{2}$ and are currently disappearing at an overall rate of 0.8 to $2 \%$ per year. ${ }^{3}$ The declining of tropical forests in different parts of the world is most probably due to the activities of human kind. ${ }^{4}$ Phytosociology is the study of the characteristics, classification, relationship and distribution of plant communities. It is useful to collect data on the quantitative change of each species studied and how their relationship with other species in the same community. Further, such studies serve as a pre-requisite for investigating the details of primary productivity of tropical ecosystems and can be used for environmental impact assessment studies in future with reference to understand the changes experienced in the past and continuing on into the future.

Most of the developed and developing countries have these basic studies and defined with the help of vegetation maps. ${ }^{5}$ Presently as the tropical forests in most part of the world are under immense anthropogenic pressure require careful management intervention to maintain their overall biodiversity and sustainability. ${ }^{6}$ The Indian subcontinent rich in biodiversity and as the 12 mega-diversity centres of the world facing problem in disappearance of tropical forests at an alarming rate due to anthropogenic activities and invasion of invasive species. ${ }^{7}$ During the period 2009 to 2011 net and gross deforestation rate in India was -0.03 and -0.43 , respectively. ${ }^{8}$ In Odisha, forest covers covering about $37.34 \%$ of the state's geographical area and about $7.66 \%$ of country's forest ${ }^{9}$ having net and gross deforestation rate during 1935 to 2010 was -0.69 and -0.79 , respectively. ${ }^{8}$ Balasore, one of the coastal districts of Odisha located at $20^{\circ} 48^{\prime}$ to $21^{\circ} 59^{\prime}$ north latitude and $86^{\circ} 16^{\prime}$ to $87^{\circ} 29^{\prime}$ 'east longitude having a total forest area of 351 sq.km consisting of 23 sq. km of dense forest, $126 \mathrm{sq} . \mathrm{km}$ of moderate dense forest and 202sq. km of open forest. ${ }^{10}$ A study mentioned that the gross deforestation rate of the district was found 0.69 with in a period of 31years from 1973 to $2004 .{ }^{8}$ As per the State of Forest Report, Govt. of India from 2003 to 2007 the comparative account of decrease in close forest cover including very dense and moderate dense forest of the district was $1.5 \mathrm{sq}$. km per year. Thus accurate quantification of vegetation especially over storey vegetation layer is a prerequisite to provide information in formulating various action plans or management plans for their restoration. In these contexts vegetation analysis pertaining to Phytosociology and community structure of over storey vegetation layer in different forest covers of Odisha was made by various workers. ${ }^{11-13}$ However, it remains as a neglected area with no such studies was made for the tropical forest covers of Balasore. Keeping paucity on quantitative information on over storey plant composition, diversity, and community characteristics the present study was designed to assess the species diversity, richness, abundance and distribution of over storey vegetation layer existing in the study area.

\section{Materials and methods}

\section{Study area}

The Phytosociological study of upper storey vegetation layer (tree) was carried out in Balasore district, Odisha. The district lies between $20^{\circ} 48^{\prime}$ to $21^{\circ} 59^{\prime}$ north latitude and $86^{\circ} 16^{\prime}$ to $87^{\circ} 29^{\prime}$ east longitude (Figure 1). The district is surrounded by Medinipur district of West Bengal in its northern side, Bay of Bengal in its east, Bhadrak district in its south and Mayurbhanj and Keonjhar districts on its western side. The climate of the district is mostly hot and humid. Annual mean temperature and precipitation of Balasore is $32^{\circ} \mathrm{C}$ and the average rain fall is $1583 \mathrm{~mm} .{ }^{14}$ Relative humidity is generally high throughout the 
year and varying in the range of $50 \%$ to $90 \%$. The district total geographical area is $3806 \mathrm{sq} \mathrm{km}$. Nearly $9.22 \%$ of the geographical area of the district is covered by forest which plays an important role in the economy of the district.

\section{Phytosociological analysis}

Upper storey vegetation layer of tropical forest covers of the district was analysed by quadrat method. A total of 42 quadrats of $20 \mathrm{~m} \times 20 \mathrm{~m}$ size were laid down at different aspects. The size and number of quadrats laid down during survey was ascertained as per species area curve ${ }^{15}$ and running mean method. ${ }^{16}$ All the tree species in quadrats were enumerated and identified following Saxena et al. ${ }^{17}$ All the trees occurring inside the quadrat were recorded and measured for girth at breast height level $(1.37 \mathrm{~m})$ from the ground. Quantitative data collected during field survey were subjected to analyze various phytosociological parameters following standard methods of calculations and formulae. ${ }^{15,16,18-20}$ The frequency values obtained were grouped in frequency classes to study the homogeneous/heterogeneous nature of vegetation. ${ }^{21}$ To assess the overall impact of a species Importance Value Index (IVI) was determined by adding relative frequency, relative density and relative basal area as per Cottam et al. ${ }^{18}$

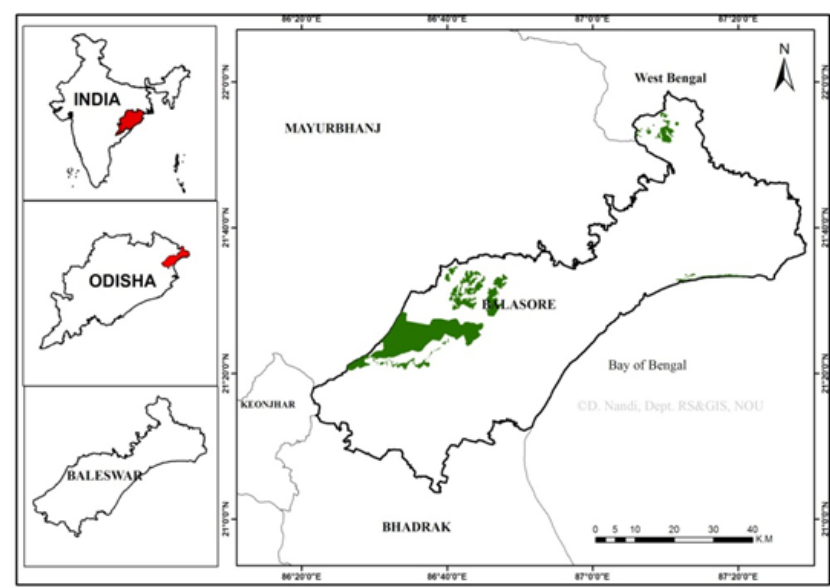

Figure I Location map of forest covers in Balasore district of Odisha.

Relative frequency (\%): Relative frequency is the degree of dispersion of individual species in an area in relation to the number of all species occurred. It was determined following the equation as

$$
\text { Relative Frequency }(\%)=(\text { Frequency of the species / Frequency of all species }) \times 100
$$

Relative Density (\%): Relative Density is the numerical strength of a species in respect to the total number of individual of all the species. It was calculated following the equation as:

$$
\text { Relative Density }=(\text { Density of the species / Density of all species }) \times 100
$$

Relative dominance (\%): Dominance is the parameter which is determined by the value of basal area. For the comparative analysis relative dominance is determined. It is the coverage value of a species with respect to the sum of coverage of the rest of the species in the area.It was calculated as:

$$
\text { Relative dominance }=(\text { Basal area of the species / Basal area of all the species }) \times 100
$$

Importance value index: Importance Value Index is used to determine the overall impact of each species in the community structure. It was calculated by the addition of the percentage values of the relative frequency, relative density and relative dominance (Relative basal area).

$$
I V I=\text { Relative dominance }(\text { Relative basal area })+\text { Relative density }+ \text { Relative frequency. }
$$

Species diversity of upper storey vegetation layer was determined with the Shannon-Wiener diversity index. ${ }^{22}$ Concentration of Dominance (CD) was calculated to evaluate the level of dominance of a species within a community and was expressed by Simpson's index. ${ }^{23}$

Shannon-wiener index (Shannon \& Wiener, 1963): It is a measure of the average degree of 'uncertainty' in predicting to what species an individual chosen at random from a collection of $\mathrm{S}$ species and $\mathrm{N}$ individuals. It was estimated by using formula:

$$
H^{\prime}=-\sum_{i=1}^{s}\left(\frac{\mathrm{ni}}{N}\right) \ln \left(\frac{\mathrm{ni}}{N}\right)
$$

Where, ni: Number of individuals belonging to the species

$\mathrm{N}$ : Total number of individuals in the sample

Dominance index (Simpson, 1949): It is a measure of dominance since it weighted towards the abundances of commonest species. It was estimated by using formula:

$$
D=\sum_{i=1}^{s}\left(\frac{\mathrm{ni}}{N}\right)^{2} \operatorname{orPi} i^{2}
$$

Where "ni" and "N" are same to Shannon-Wiener index.

\section{Results and discussion}

\section{Floristic composition}

A total of 94 species belonging to 77 genera and 38 families were recorded from the study area. A majority of the families were represented by only one or two species (Table 1). The most common families were Euphorbiaceae (10species) and Rubiaceae (8species) followed by Combretaceae ( 5 species), Caesalpiniaceae=Ebenace$\mathrm{ae}=$ Anacardiacea $=$ Apocynaceae $=$ Verbenaceae $=$ Oleaceae $\quad(4$ species each), Rutaceae $=$ Barringtoniaceae $(3$ species each), etc. The number of species per genus was more than 1.2 and that per family was nearly equal to 2.5. The species richness of 94species over 16.8ha area of the district reflects a moderate level of diversity in forests of Eastern Ghats (Table 1). The results of the study compared well to other inventories conducted in tropical forests both in India and elsewhere. Murphy and Lugo ${ }^{24}$ emphasized that average tree species richness of a dry tropical forest community ranges from 35 to 90 . Condit ${ }^{25}$ recorded 63 species from 50ha plot at Mudumalai Forest Reserve, India to 996 species in 52ha plot at Lambir, Malaysia. In a recent assessment of species richness in southern Eastern Ghats, Pragasan and Parthasarathy ${ }^{26}$ recorded 272 species in 60ha sampled area. 
Table I Species composition, frequency (\%), density (Plants/ ha), basal area ( $\left.\mathrm{m}^{2} / \mathrm{ha}\right), \mathrm{A} / \mathrm{F}$ ratio, importance value index (IVI), species diversity ( $\left.\mathrm{H}^{\prime}\right)$ and concentration of dominance $(\mathrm{Cd})$ of over storey vegetation layer in tropical forests of Balasore district

\begin{tabular}{|c|c|c|c|c|c|c|c|c|}
\hline Name of the Species & Family & $\begin{array}{l}\text { Density } \\
\text { (Plants/ha) }\end{array}$ & $\begin{array}{l}\text { Frequency } \\
(\%)\end{array}$ & $\begin{array}{l}\text { Basal Area } \\
\text { (m2/ha) }\end{array}$ & $\mathbf{A} / \mathbf{F}$ & IVI & $\begin{array}{l}\text { Species } \\
\text { Diversity(H') }\end{array}$ & $\begin{array}{l}\text { Concentration of } \\
\text { Dominance (Cd) }\end{array}$ \\
\hline Acacia leucophloea (Roxb.) Willd & Mimosaceae & 2.38 & 9.52 & 0.009 & 0.049 & 1.13 & 0.019 & 0.000011 \\
\hline Acacia pennata ( L.)Willd. & Mimosaceae & 1.19 & 4.76 & 0.144 & 0.11 & 1.05 & 0.01 & 0.00003 \\
\hline Actinodaphne angustifolia (BI.) Nees & Lauraceae & 4.76 & 9.52 & 0.1 & 0.1 & $1.8 \mathrm{I}$ & 0.033 & 0.00004 \\
\hline Aegle marmelos (L.) Corr. & Rutaceae & 2.38 & 9.52 & 0.096 & 0.049 & 1.43 & 0.019 & 0.000011 \\
\hline Alangium salvifolium (L.f.)Wang. & Alangiaceae & 1.19 & 4.76 & 0.053 & 0.11 & 0.74 & 0.01 & 0.000003 \\
\hline Alstonia scholaris (L.) R. Br. & Apocynaceae & 2.38 & 4.76 & 0.2 & 0.2 & $1.4 \mathrm{I}$ & 0.019 & 0.000011 \\
\hline $\begin{array}{l}\text { Anogeissus latifolia (Roxb.ex.Dc.) } \\
\text { Wall.exGuill. \&Perr. }\end{array}$ & Combretaceae & 9.52 & 33.33 & 0.233 & 0.02 & 4.82 & 0.057 & 0.0002 \\
\hline $\begin{array}{l}\text { Anthocephalus chinensis (Lam.)A. } \\
\text { Rich. ex Walp. }\end{array}$ & Rubiaceae & 4.76 & 9.52 & 0.1 & 0.1 & 1.91 & 0.033 & 0.00004 \\
\hline Antidesma ghasembilla Gaertn. & Euphorbiaceae & 1.19 & 4.76 & 0.03 & 0.1 & 0.66 & 0.01 & 0.000003 \\
\hline $\begin{array}{l}\text { Aporusa octandra (Buch.-Ham.exD. } \\
\text { Don) }\end{array}$ & Euphorbiaceae & 1.19 & 4.76 & 0.007 & 0.1 & 0.57 & 0.011 & 0.000003 \\
\hline Barringtonia acutangula (L.) Gaertn. & Barringtoniaceae & 1.19 & 4.76 & 0.13 & 0.1 & 1.02 & 0.011 & 0.000003 \\
\hline Bauhinia malabarica Roxb. & Caesalpiniaceae & 1.19 & 4.76 & 0.174 & 0.1 & 1.16 & 0.011 & 0.000003 \\
\hline Bauhinia purpurea L. & Caesalpiniaceae & 1.19 & 4.76 & 0.019 & 0.1 & 0.62 & 0.011 & 0.000003 \\
\hline Bauhinia semla Wunderl & Caesalpiniaceae & 2.38 & 4.76 & 0.203 & 0.2 & 1.42 & 0.018 & 0.000011 \\
\hline Bombax ceiba L. & Bombacaceae & 9.52 & 19.05 & 0.631 & 0.049 & 5.05 & 0.057 & 0.000171 \\
\hline Boswellia serrata Roxb.ex Colebr. & Burseracee & 25 & 33.33 & 1.16 & 0.04 & 10.18 & 0.116 & 0.00118 \\
\hline Bridelia airy-shawii P.T.Li & Euphorbiaceae & 3.571 & 14.29 & 0.23 & 0.03 & 2.45 & 0.026 & 0.000024 \\
\hline Buchanania lanzan Speng. & Anacardiaceae & 22.62 & 28.57 & 0.375 & 0.049 & 6.73 & 0.107 & 0.000964 \\
\hline $\begin{array}{l}\text { Canthium dicoccum (Gaertn.)Teijsm } \\
\text { \& Binnend. }\end{array}$ & Rubiaceae & 3.57 & 9.52 & 0.041 & 0.07 & $\mathrm{I} .4$ & 0.026 & 0.000024 \\
\hline Careya arborea Roxb. & Barringtoniaceae & 7.14 & 23.81 & 0.07 & 0.025 & 3.15 & 0.045 & 0.0001 \\
\hline Casearia graveolens Dalz & Flacourtiaceae & 4.76 & 19.05 & 0.019 & 0.02 & 2.26 & 0.033 & 0.000043 \\
\hline Cassia fistula L. & Caesalpiniaceae & 7.14 & 19.05 & 0.059 & 0.08 & 2.73 & 0.045 & 0.00001 \\
\hline $\begin{array}{l}\text { Chionanthus macrophylla (Wall.ExG. } \\
\text { Don)Bl. }\end{array}$ & Oleaceae & 5.95 & 4.76 & 0.076 & 0.5 & 1.47 & 0.039 & 0.00007 \\
\hline $\begin{array}{l}\text { Chionanthus mala- elengi (Dennst.) } \\
\text { Green }\end{array}$ & Oleaceae & 8.33 & 14.29 & 0.264 & 0.08 & 3.22 & 0.051 & 0.000131 \\
\hline $\begin{array}{l}\text { Cleistanthus collinus (Roxb.) Benth. } \\
\text { ex. Hook.f. }\end{array}$ & Euphorbiaceae & 8.33 & 14.29 & 0.131 & 0.08 & 2.76 & 0.051 & 0.000131 \\
\hline Combretum roxburghii Spreng. & Combretaceae & 1.19 & 4.76 & 0.001 & 0.11 & 0.56 & 0.01 & 0.000003 \\
\hline Croton roxburghii Balak & Euphorbiaceae & 25 & 38.09 & 0.493 & 0.03 & 8.23 & 0.116 & 0.001177 \\
\hline Cycas circinalis L. & Cycadaceae & 1.19 & 4.76 & 0.01 & 0.1 & 0.59 & 0.01 & 0.000003 \\
\hline Dalbergia lanceolaria L.f. & Moraceae & 2.38 & 4.76 & 0.29 & 0.2 & $\mathrm{I} .73$ & 0.018 & $0.00001 \mathrm{I}$ \\
\hline Dalbergia latifolia Roxb. & Fabaceae & 2.38 & 9.52 & 0.23 & 0.049 & 1.9 & 0.019 & $0.00001 \mathrm{I}$ \\
\hline Dalbergia volubilis Roxb. & Fabaceae & 1.19 & 4.76 & 0.002 & 0.1 & 0.56 & 0.01 & 0.000003 \\
\hline Dillenia pentagyna Roxb. & Dilleniaceae & 11.91 & 23.81 & 1.299 & 0.04 & 8.1 & 0.067 & 0.0003 \\
\hline Diospyros sylvatica Roxb. & Ebenaceae & 4.76 & 14.29 & 0.037 & 0.049 & 1.94 & 0.033 & 0.000043 \\
\hline Diospyros malabarica (Desr.) Kostel & Ebenaceae & 5.95 & 9.52 & 2.215 & 0.13 & 9.33 & 0.039 & 0.00007 \\
\hline Diospyros melanoxylon Roxb. & Ebenaceae & 17.86 & 23.81 & 0.116 & 0.06 & 4.78 & 0.09 & 0.000601 \\
\hline Diospyros montana Roxb. & Ebenaceae & 7.14 & 14.29 & 0.122 & 0.07 & 2.56 & 0.045 & 0.0001 \\
\hline
\end{tabular}


Table Continued.

\begin{tabular}{|c|c|c|c|c|c|c|c|c|}
\hline Name of the Species & Family & $\begin{array}{l}\text { Density } \\
\text { (Plants/ha) }\end{array}$ & $\begin{array}{l}\text { Frequency } \\
\text { (\%) }\end{array}$ & $\begin{array}{l}\text { Basal Area } \\
\text { (m2/ha) }\end{array}$ & $\mathbf{A} / \mathbf{F}$ & IVI & $\begin{array}{l}\text { Species } \\
\text { Diversity(H') }\end{array}$ & $\begin{array}{l}\text { Concentration of } \\
\text { Dominance (Cd) }\end{array}$ \\
\hline Erhetia laevis Roxb. & Ehretiaceae & 1.19 & 4.761 & 0.001 & 0.1 & 0.56 & 0.011 & 0.000003 \\
\hline $\begin{array}{l}\text { Fagerlindia fasciculate (Roxb.) } \\
\text { Tirveng. }\end{array}$ & Rubiaceae & 9.52 & 28.57 & 0.2 & 0.02 & 4.33 & 0.057 & 0.000171 \\
\hline Garuga pinnata Roxb. & Burseracee & 1.19 & 4.76 & 0.014 & 0.1 & 0.59 & 0.01 & 0.000003 \\
\hline Glochidion lanceolarium (Roxb.) Dalz & Euphorbiaceae & 16.66 & 19.05 & 0.196 & 0.09 & 4.51 & 0.086 & 0.000523 \\
\hline Grewia tilifolia Vahl. & Tiliaceae & 1.19 & 4.76 & 0.011 & 0.1 & 0.59 & 0.01 & 0.000003 \\
\hline Haldinia cordifolia (Roxb.)Ridsd. & Rubiaceae & 8.33 & 19.05 & 0.468 & 0.04 & 4.32 & 0.051 & 0.000131 \\
\hline Heterophragma roxburghii L. & Bignoniaceae & 1.19 & 4.76 & 0.007 & 0.1 & 0.57 & 0.01 & 0.000003 \\
\hline $\begin{array}{l}\text { Holarrhena pubescens (Buch-Ham.) } \\
\text { Wall.ex G Don }\end{array}$ & Apocynaceae & 19.05 & 57.14 & 0.285 & 0.01 & 8.23 & 0.095 & 0.000683 \\
\hline Holoptelia integrifolia (Roxb.)Planch. & Ulmaceae & 1.19 & 4.76 & 0.016 & 0.1 & 0.6 & 0.01 & 0.000003 \\
\hline Ixora pavetta Andr. & Rubiaceae & 2.38 & 4.76 & 0.011 & 0.21 & 0.75 & 0.019 & 0.000011 \\
\hline Ixora undulate Roxb. & Rubiaceae & 2.38 & 9.52 & 0.048 & 0.049 & 1.26 & 0.019 & 0.000011 \\
\hline Kydia calycina Roxb. & Malvaceae & 2.38 & 9.52 & 0.261 & 0.049 & 2.01 & 0.019 & 0.000011 \\
\hline Lagerstroemia paviflora Roxb. & Lythraceae & 3.57 & 9.52 & 0.037 & 0.07 & 1.39 & 0.026 & 0.000024 \\
\hline Lannea coromandelica (Houtt.)Nerr. & Anacardiaceae & 7.14 & 14.29 & 0.124 & 0.07 & 2.57 & 0.045 & 0.0001 \\
\hline Leea asiatica (L.) Ridsdale & Vitaceae & 1.19 & 4.76 & 0.008 & 0.1 & 0.58 & 0.01 & 0.000003 \\
\hline Ligustrum gamblei Ramam. & Oleaceae & 1.19 & 4.76 & 0.01 & 0.1 & 0.6 & 0.01 & 0.000003 \\
\hline Limonia crenulata Roxb. & Rutaceae & 1.19 & 4.76 & 0.036 & 0.1 & 0.68 & 0.01 & 0.000003 \\
\hline Litsea glutinosa (Lour) Robins. & Lauraceae & 1.19 & 4.7619 & 0.025 & 0.1 & 0.64 & 0.011 & 0.000003 \\
\hline Litsea monopetala (Roxb.) Pers. & Lauraceae & 1.19 & 4.76 & 0.024 & 0.1 & 0.63 & 0.011 & 0.000003 \\
\hline $\begin{array}{l}\text { Macaranga peltata (Roxb.)Muell.- } \\
\text { Arg. }\end{array}$ & Euphorbiaceae & 9.52 & 33.33 & 0.062 & 0.02 & 4.22 & 0.057 & 0.000171 \\
\hline Madhuca indica Gmel. & Sapotaceae & 21.43 & 14.29 & 0.232 & 0.2 & 4.91 & 0.104 & 0.000865 \\
\hline $\begin{array}{l}\text { Mallatous philippensis (Lam.) Muell.- } \\
\text { Arg. }\end{array}$ & Euphorbiaceae & 1.19 & 4.76 & 0.005 & 0.1 & 0.57 & 0.011 & 0.000003 \\
\hline Mangifera indica L. & Anacardiaceae & 8.33 & 9.52 & 5.451 & 0.18 & 20.98 & 0.051 & 0.000131 \\
\hline Melia azadirachta L. & Meliaceae & 1.19 & 4.76 & 0.045 & 0.1 & 0.71 & 0.01 & 0.000003 \\
\hline Meyna spinosa Roxb.ex Link. & Rubiaceae & 1.19 & 4.76 & 0.011 & 0.1 & 0.588 & 0.011 & 0.000003 \\
\hline $\begin{array}{l}\text { Miliusa velutina (Dunal) Hook.f.\& } \\
\text { Thoms. }\end{array}$ & Annonaceae & 1.19 & 4.76 & 0.001 & 0.1 & 0.54 & 0.011 & 0.000003 \\
\hline Morinda pubescens Sm. & Rubiaceae & 1.19 & 4.76 & 0.002 & 0.1 & 0.56 & 0.011 & 0.000003 \\
\hline Murraya paniculata (L.)Jack. & Rutaceae & 2.38 & 4.76 & 0.009 & 0.21 & 0.74 & 0.019 & 0.000011 \\
\hline Nyctanthes arbor-tristis L. & Oleaceae & 1.19 & 4.76 & 0.018 & 0.1 & 0.61 & 0.011 & 0.000003 \\
\hline Ochna squarrosa L. & Ochnaceae & 1.19 & 4.76 & 0.006 & 0.1 & 0.57 & 0.01 & 0.000003 \\
\hline Oroxylum indicum (L.) Vent & Bignoniaceae & 3.57 & 14.29 & 0.086 & 0.03 & 1.95 & 0.026 & 0.000024 \\
\hline Phyllanthus emblica L. & Euphorbiaceae & 3.57 & 9.52 & 0.03 & 0.07 & 1.36 & 0.026 & 0.000024 \\
\hline Polyalthia suberosa L. & Annonaceae & 1.19 & 4.76 & 0.021 & 0.1 & 0.62 & 0.01 & 0.000003 \\
\hline Pongamia pinnata (L.)Pierre & Fabaceae & 19.05 & 19.05 & 1.721 & 0.1 & 10.17 & 0.095 & 0.000683 \\
\hline Premna calycina Haines & Verbenaceae & 5.95 & 4.76 & 0.174 & 0.5 & 1.81 & 0.039 & 0.00007 \\
\hline Pterocarpus marsupium Roxb. & Fabaceae & 13.09 & 23.81 & 0.231 & 0.04 & 4.53 & 0.072 & 0.000323 \\
\hline Pterospermum acerifolium (L.)Willd & Sterculiaceae & 1.19 & 4.76 & 0.041 & 0.1 & 0.69 & 0.01 & 0.000003 \\
\hline Schleichera oleosa (Lour.) Oken & Sapindaceae & 14.29 & 28.57 & 0.31 & 0.03 & 5.35 & 0.077 & 0.000384 \\
\hline Shorea robusta Gaertn.f & Dipterocarpaceae & 134.523 & 66.66 & 4.753 & 0.06 & 40.47 & 0.312 & 0.034092 \\
\hline Soymida febrifuge (Roxb.)A. Juss. & Meliaceae & 3.57 & 4.76 & 0.04 & 0.3 & 1.03 & 0.026 & 0.000024 \\
\hline
\end{tabular}

Citation: Mishra RK, Parhi S, Biswal AK. Diversity of over storey plant communities of tropical forest covers of Balasore district, Odisha, India. Adv Plants Agric Res. 2018;8(I):20-26. DOI: I0.I5406/apar.2018.08.00285 
Table Continued.

\begin{tabular}{|c|c|c|c|c|c|c|c|c|}
\hline Name of the Species & Family & $\begin{array}{l}\text { Density } \\
\text { (Plants/ha) }\end{array}$ & $\begin{array}{l}\text { Frequency } \\
(\%)\end{array}$ & $\begin{array}{l}\text { Basal Area } \\
\text { (m2/ha) }\end{array}$ & $\mathbf{A} / \mathbf{F}$ & IVI & $\begin{array}{l}\text { Species } \\
\text { Diversity(H') }\end{array}$ & $\begin{array}{l}\text { Concentration of } \\
\text { Dominance (Cd) }\end{array}$ \\
\hline Spondias pinnata (L.f.)Kurz & Anacardiaceae & 1.19 & 4.76 & 0.001 & 0.1 & 0.57 & 0.01 & 0.000003 \\
\hline $\begin{array}{l}\text { Sterospermum colais (Buch.-Ham.ex } \\
\text { Dillw.) Mabberley }\end{array}$ & Bignoniaceae & 1.19 & 4.76 & 0.001 & 0.1 & 0.59 & 0.011 & 0.000003 \\
\hline Strychnos nox-vomica L. & Strychnaceae & 2.38 & 4.76 & $0.04 I$ & 0.21 & 0.86 & 0.019 & 0.000011 \\
\hline Syzygium cumini (L.)Skeels & Myrtaceae & 13.09 & 42.86 & 0.491 & 0.01 & 6.97 & 0.072 & 0.000323 \\
\hline $\begin{array}{l}\text { Syzygium cerasoides (Roxb.) Chatt. } \\
\text { \& Kanjilal f. }\end{array}$ & Myrtaceae & 1.19 & 4.76 & 0.144 & 0.1 & 1.06 & 0.01 & 0.000003 \\
\hline Tectona grandis L.f. & Verbenaceae & 3.57 & 4.76 & 0.028 & 0.3 & 0.97 & 0.026 & 0.000024 \\
\hline Terminalia alata Heyne.ex Roth & Combretaceae & 70.24 & 61.91 & 1.496 & 0.03 & 19.87 & 0.225 & 0.009294 \\
\hline Terminalia bellirica (Gaertn.) Roxb. & Combretaceae & 10.71 & 23.81 & 0.37 & 0.04 & 4.71 & 0.062 & 0.000216 \\
\hline Terminalia chebula Retz. & Combretaceae & 3.57 & 9.52 & 0.063 & 0.07 & $\mathrm{I} .48$ & 0.026 & 0.000024 \\
\hline Trema orientalis (L.) BI. & Ulmaceae & 2.38 & 4.76 & 0.04 & 0.2 & 0.88 & 0.019 & 0.000011 \\
\hline Trewia nudiflora L. & Euphorbiaceae & 4.76 & 9.52 & 0.245 & 0.11 & 2.28 & 0.033 & 0.00004 \\
\hline Vitex peduncularis Wall.ex Schauer & Lamiaceae & 1.19 & 4.76 & 0.021 & 0.1 & 0.62 & 0.01 & 0.000003 \\
\hline Vitex pinnata L. & Verbenaceae & 3.57 & 9.52 & 0.159 & 0.07 & 1.82 & 0.026 & 0.000024 \\
\hline Vitex trifolia L. & Verbenaceae & 1.19 & 4.76 & 0.252 & 0.1 & 1.43 & 0.011 & 0.000003 \\
\hline Wendlandia tinctoria (Roxb.) DC & Rubiaceae & 7.14 & 14.29 & 0.122 & 0.07 & 2.56 & 0.045 & 0.0001 \\
\hline Wrightia arborea (Dennst.)Mabb. & Apocynaceae & 9.52 & 14.29 & 0.119 & 0.09 & 2.88 & 0.056 & 0.000171 \\
\hline Wrightia tinctoria (Roxb.) R.Br. & Apocynaceae & 14.29 & 23.81 & 0.113 & 0.049 & 4.28 & 0.077 & 0.000384 \\
\hline \multirow{2}{*}{ Xantolis tomentosa (Roxb.)Rafin. } & \multirow{2}{*}{ Sapotaceae } & 20.24 & 28.57 & 0.347 & 0.049 & 6.3 & 0.099 & 0.000772 \\
\hline & & 728.474 & & 28.453 & & 300 & 3.681 & 0.055475 \\
\hline
\end{tabular}

\section{Distribution pattern of species}

Of the 94 tree species recoded in the study area most of the species exhibited contiguous distribution (70 species). Only few species exhibited random (17species) and regular (7species) distribution. Contiguous distribution was more pronounced by Chionanthus macrophylla, Premna calycina, Soymida febrifuge, Tectona grandis, Ixora pavetta, Murraya paniculata, Strychnos nox-vomica, etc. Species showed regular distribution were Anogeissus latifolia, Macaranga peltata, Holarrhaena pubescens, Careya arborea, Fragerlindia fasciculate, Syzygium cumini and Casearia graveolens (Table 1).

\section{Raunkiaer's frequency class distribution}

The over storey species had a wide range of occurrence in the study site ranging in frequency from 4.76 to $66.67 \%$ and most of the species occurred only twice (Table 1). The distribution of the species into Raunkiaer's frequency classes showed that most of the species encountered were in class " $A$ " followed by class " $B$ " and equal in "C" and "D" (Figure 2). This indicates that most of the species had low frequency as would be expected in typical species-abundance distribution in tropical forests. ${ }^{27}$ Raunkiaer $^{21}$ frequency distribution holds good for this forest cover also. However this forest had only four classes instead of five as described in the law. This was due to its higher heterogeneous nature and deviation from the normal frequency distribution as described by Raunkiaer. ${ }^{21}$

\section{Stand structure}

Structural parameters like density (plants/ha), basal area $\left(\mathrm{m}^{2} / \mathrm{ha}\right)$ and frequency (\%) of upper-storey vegetation layer of the study area is presented in Table 1. Species wise density of individuals having

$\geq 30 \mathrm{~cm}$ girth ranged from 2 to 134 plants/ha and the total density of 729 plants/ha. Maximum density was recorded for Shorea robusta (134) followed by Terminalia alata (70), Croton roxburghii (25), Boswellia serrata (25), Buchnania lanzan (22.62), Madhuca indica (21.43) and Xantolish tomentosa (20.24). The minimum density of less than equal to two was observed for many species like Aporusa octandra, Spondias pinnata, Miliusa velutina, Bauhinia purpurea, Sterospermum colais, Litsia monopetala, Vitex penducularis, Syzygium cerasoides, etc. Rest of the species showed intermediate range of density per hectare

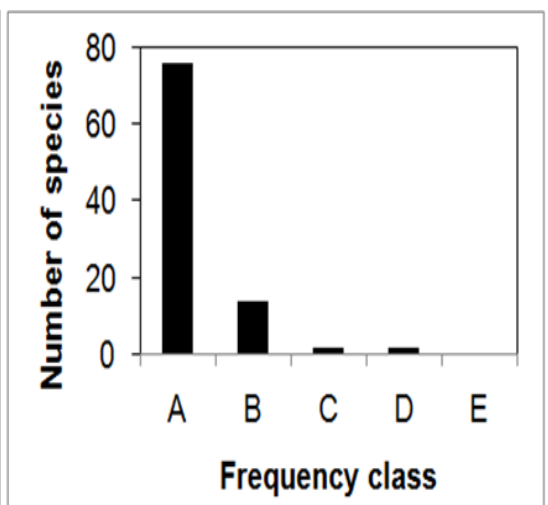

Figure 2 Raunkiaer's frequency class distribution of over storey vegetation layer of the study area.

The tree density of 729 individuals/ha recorded in the present investigation is lower as compared to densities reported from Saddle Peak of North Andaman Islands and Great Andaman Groups (946-1137trees/ha, $\left.{ }^{28}\right]$ and well comparable to tropical forests of 
Kalkad, Western Ghats $\left(575-855\right.$ trees/ha, $\left.{ }^{29}\right)$, Similipal, Odisha, ${ }^{30,31}$ Brazil (420-777trees/ha, ${ }^{32}$ ), seasonally deciduous forest of Central Brazil (734trees/ha, ${ }^{33}$ ), Semi deciduous forest of Piracicaba, Brazil $\left(842\right.$ trees $/$ ha,$\left.{ }^{34}\right)$ and Costa Rica $\left(617\right.$ trees/ha, $\left.{ }^{35}\right)$. Basal area of upper storey vegetation in the study area ranged from $0.001-5.451 \mathrm{~m}^{2} /$ ha, the highest for Mangifera indica and lowest for many species like Ethetia laevis, Sterospermum colais, Spondias pinnata and Miliusa velutina (Table 1). Basal areas for some important timber species such as Shorea robusta, Tectona grandis and Dalbergia lanceolaria were 4.753 , 0.028 and $0.290 \mathrm{~m}^{2} /$ ha, respectively. The overall basal area estimated was $28.453 \mathrm{~m}^{2} /$ ha. Mangifera indica contributed maximum of $19 \%$ to the total basal area followed by Shorea robusta $(16.61 \%)$, Diospyros malabarica (7.72\%) and Pongamia pinnata (6\%). The total contribution that resulted from this associated combination of Mangifera-Shorea-Diospyros-Pongamia was $50 \%$. The overall basal area estimated of upper storey vegetation layer is well within the reported range of various Indian tropical forests ${ }^{36}$ and lower than the value reported from Monteverde of Costa Rica $\left(62 \mathrm{~m}^{2} / \mathrm{ha},{ }^{37}\right)$. High basal area is a characteristic feature of mature forest stand and serves as a reflection of high performance of the trees. It may also presuppose the development of an extensive root system used efficient nutrient absorption, growth suppressing of subordinate plants as they intercept much of the solar radiation that might otherwise reach the forest floor.

\section{Ecological importance of species}

Importance Value Index (IVI) is the measurement of relative contribution of a species to the entire community and suggesting the ability of a species to establish over an array of habitats. However, there is no single perfect way of assessing the relative contribution of a species. The abundance of a species can be represented by several measures such as relative density, relative frequency and Importance Value Index (IVI). Though frequency and density values are suitable for herbs and shrubs, ${ }^{39} \mathrm{IVI}$ is an important information for tree species. On the basis of IVI, Shorea robusta was found as the dominant species having IVI of 40.47 followed by Mangifera indica (20.98), Terminalia alata (19.87), Boswellia serrata (10.18), Pongamia pinnata (10.17), etc. Miliusa velutina had IVI of 0.054 was considered as the rare species of the study area (Table 1). All other tree species showed intermediate range of IVI. High IVI values exhibited by those species clearly indicate the ecological importance of corresponding species. Such measurement of over storey vegetation layer also helps in understanding the ecological significance of a species in its community/habitat. Higher is the IVI more ecological significance of the species in a particular ecosystem. ${ }^{27}$ Furthermore, information of IVI would of prime importance in deciding the management options for specific host population of native wildlife that is facing the danger of local extinction due to heavy human pressure surrounding this forest cover.

\section{Diversity measures}

Species diversity and concentration of dominance of upper-storey vegetation layer of the study area is given in Table 1. Measurement of biodiversity of specific area (local scale) on the basis of species richness does not provide a complete understanding about the individuals of the species in an ecosystem as it suffers from the lack of evenness or equitability. Shannon Wiener's index of diversity is one of the popular measures of species diversity. It ranged from 0.01 to 0.312 across the study area with a total diversity value of 3.68. Maximum species diversity of 0.312 was experienced by Shorea robsusta while the minimum of 0.01 was experienced by many species of the study area indicating that over storey vegetation layer of Balasore was highly diverse. The species diversity is generally higher for tropical forests, which is reported as 5.06 and 5.40 for young and old stand, respectively. ${ }^{39}$ For Indian forests the diversity index ranges between 0.83-4.1. ${ }^{41}$ Higher species diversity index in tropical forests as reported by $\mathrm{Knight}^{40}$ in comparison to the present investigation may be due to differences in the area sampled and lack of uniform plot dimensions. In contrast to species diversity the concentration of dominance of such vegetation layer ranged from 0.000003 to 0.03 . Maximum value was experienced by Shorea robusta and minimum by many species (Table 1). The range of concentration of dominance estimated for over-storey vegetation layer of the district implies that most of the species are equitably distributed while very few species showed the degree of dominance ${ }^{42}$ The range of concentration of dominance estimated of the study area is less than those recorded in Nelliampathy $\left(0.085 ;{ }^{43}\right)$ and tropical dry deciduous forests of Western India $(0.08-0.16 ; 44)$ and indicates the absence of single species dominance.

\section{Conclusion}

Tropical forest covers of Balasore district supports a diverse plant community. The rich plant diversity is worthy for its conservation to check it from further reduction in species richness, rapid deforestation and forest fragmentation. The rare species of the area identified based on IVI must need proper attention to determine their conservation status and key functions. Further research on mapping of such species with respect to their concentrated distribution in some pockets of the study area and study of their key ecological and structural functions would help to identify locations for conservation actions.

\section{Acknowledgements}

The authors are thankful to the Divisional Forest Officer (DFO), Balasore Wildlife Division and their staff for their help rendered during the study period to carry out the research work.

\section{Conflict of interest}

The author declares no conflict of interest.

\section{References}

1. Myers N. Two key challenges for biodiversity:discontinuities and synergisms. Biodiversity Conservation. 1996;5(9):1025-1034.

2. Wilson EO. 1992) The Diversity of Life. Belknap, Cambridge, Massachusetts.

3. Sagar R, Raghubanshi AS, Singh JS (2003) Tree species composition, dispersion and diversity along a disturbance gradient in a dry tropical forest region of India. Forest Ecology and Management. 1992;186:6171.

4. FAO (Food and Agriculture Organization). The State of Food and Agriculture, Rome, Italy; 2005.

5. Naidu MT, Kumar OA. Tree diversity, stand structure, and community composition of tropical forests in Eastern Ghats of Andhra Pradesh, India. Journal of Asia-Pacific Biodiversity. 2016;9(3):328-334.

6. Yam G, Tripathi OP. Tree diversity and community characteristics in Talle Wildlife Sanctuary, Arunachal Pradesh, Eastern Himalaya, India. Journal of Asia-Pacific Biodiversity. 2016;9(2):160-165.

7. Ramachandran A, Radhapriya P, Jayakumar S, et al. Critical analysis of forest degradation in the southern eastern ghats of India:comparison of satellite imagery and soil quality index. PLOS ONE. 2016;11(1):1-19.

8. Reddy CS, Dutta K, Jha CS. Analysing the gross and deforestation rates in India. Current Science. 2013;105(11):1492-1500. 
9. SPCB. Orissa: State of environment Orissa. Member Secretary, State Pollution Control Board, Orissa; 2006.

10. FSI (Forest Survey of India). Tree Cover. India State of Forest Report, Dehradun, India; 2015.

11. Mishra RK, Upadhyay VP, Mohanty RC. Vegetation ecology of the Similipal biosphere reserve, Orissa, India. Journal of Applied Ecology and Environmental Research. 2008;6(2):89-100.

12. Mishra RK, Upadhyay VP, Mishra PK, et al. Ecological problems of tree species in two protected ecosystems of Eastern Ghats, India. Journal of Environmental Biology. 2011;32(1):115-119.

13. Mishra RK, Upadhyay VP, PattanaikS, et al. Composition and stand structure of tropical moist deciduous forest of Similipal Biosphere Reserve. Orissa, India. Croatia: Intech Books; 2012.

14. www.yr.no./place/India/Orissa/Balasore/Statistics.html

15. Misra R. Ecology Work Book. New Delhi, India: Oxford and IBH Publishing Co; 1968.

16. Kershaw KR. Quantitative and Dynamic Plant Ecology. London: Edward Arnold Ltd; 1973.

17. Saxena HO, Brahmam M. The Flora of Orissa. Vol. I-IV regional research laboratory (CSIR), Bhubaneswar, India: Bhubaneswar and Orissa Forest Development Corporation Ltd; 1996.

18. Cottom G, Curtis JT. The use of distance measures in the phytosociological sampling. Ecology. 1956;37(3):451-460.

19. Tüxen R. Die heutigepotentiellenatürliche vegetation also Gegenstandder vegetationskartierung. Angew Pflanz. Stolzenau, Germany. 1956;13:4-42.

20. Curtis JT. The Vegetation of Wisconsin, An Ordination of Plant Communities. Madison. Wisconsin, USA: University Wisconsin Press; 1959.

21. Raunkiaer C. The Life Forms of Plants and Statistical Plant Geography. UK: Oxford University Press; 1934.

22. Shannon CE, Wiener W. The Mathematical Theory of Communication. Illinois, USA: University Press; 1963.

23. Simpson EH. Measurement of diversity. Nature. 1949;163:688.

24. Murphy PG, Lugo AE. Ecology of tropical dry forest. Annual Review of Ecology and Systematics. 1986;17:67-88.

25. Condit R, Ashton PS, Baker P, et al. Spatial pattern in the distribution of tropical tree species. Science. 2000;288(5470):1414-1418.

26. Pragasan LA, Parthasarathy N. Landscape-level tree diversity assessment in tropical forests of southern Eastern Ghats, India. Flora. 2010;205(11):728-737.

27. Odum EP. Fundamentals of Ecology. Philadelphia: WB Saunders Co; 1971.

28. Padalia H, Chauhan N, Porwal MC. Phytosociological observations on tree species diversity of Andaman Islands, India. Current Science. 2004;87(6):799-806.

29. Kadavul K, Parthasarathy N. Structure and composition of woody species in tropical semievergreen forest of Kalayan hills, Eastern Ghats, India. Tropical Ecology. 1999;40:247-260.
30. Mishra RK, Pattanaik S, Mohanty RC. Effect of disturbance on seed germination and seedling growth of Cassia fistula (L.), Albizia lebbeck (L.) Benth. And Dalbergiasissoo Roxb. Of Similipal Biosphere Reserve, Odisha, India. Forest Res. 2014;3:130.

31. Pattanaik S, Dash A, Mishra RK, et al. Seed germination and seedling survival percentage of Shorea robusta Gaertn.f. in buffer areas of Similipal Biosphere Reserve, Odisha, India. Journal Ecosystem Ecography. 2015;5(1):1-4

32. Campbell DG, Stone JL, Rosas A. A comparison of the Phytosociology and dynamics of three floodplain (Varzea) forest of known ages, Rio Jurua, western Brazilian Amazon. Botanical Journal of the Linnean Society. 1992;108:231-237.

33. Felfili JM, André R, Nascimento T, et al. Floristic composition and community structure of a seasonally deciduous forest on limestone outcrops in Central Brazil. Revista Brasil Botany. 2007;30(4):611-621.

34. Viana VM, Tabanez AAJ. Biology and conservation of forest fragments in the Brazilian Atlantic Moist Forest. In Forest patches in tropical landscapes. J Schelas, et al. editors. Washington, USA: Island Press; 1996.

35. Heaney A, Proctor J. Preliminary studies on forest structure and floristics on volcan Barva, Costa Rica. Journal Tropical Ecology. 1990;6:307320 .

36. Sapkota IP, Tigabu M, Oden PC. Spatial distribution, advanced regeneration and stand structure of Nepalese Sal (Shorea robusta) forests subject to disturbances of different intensities. Forest Ecology and Management. 2009;257:1966-1975.

37. Nadkarni NMT, Matelson J, Haber WA. Structural characteristics and florist composition of a neo tropical cloud forest. Monteverde, Costa Rica. Journal Tropical Ecology. 1995;11:484-495.

38. Ludwig JA, Reynolds JF. Statistical Ecology. New York: Wiley \& Sons; 1998.

39. Airi S, Rawal RS, Dhar U, et al. Assessment of availability and habitat preference of Jatamansi: a critically endangered medicinal plant of west Himalaya. Current Science. 2000;79(10):1467-1470.

40. Knight DH. Aphytosociological analysis of species rich tropical forest on Barro Colorado Island, Panama. Ecological Monographs. 1975;45(3):259-289.

41. Visalakshi N. Vegetation analysis of two tropical dry evergreen forests in southern India. Tropical Ecology. 1995;36:117-127.

42. Pascal JP, Pellissier R. Structure and floristic composition of a tropical evergreen forest in south west India. Journal of Tropical Ecology. $1996 ; 12: 190-216$.

43. Chandrashekara UM, Ramakrishnan PS. Vegetation and gap dynamics of a tropical wet evergreen forest in the Western Ghats of Kerala, India. Journal of Tropical Ecology. 1994;10(3):337-354.

44. Kumar, JIN, Sajish PR, Kumar RN, et al. Wood and leaf litter decomposition and nutrient release from Tectonagrandis Linn. F. in a tropical dry deciduous forest of Rajasthan, Western India. Journal of Forest Science. 2010;26(1):17-23. 